\title{
Analizando la enseñanza de liderazgo en la formación del enfermero en Brasil.
}

\author{
Gisleangela Lima Rodrigues Carrara ${ }^{1}$ \\ Jorge Luiz Rigobello ${ }^{2}$ \\ Paula Batista Luize ${ }^{3}$ \\ Priscila Lapaz Baldo ${ }^{4}$ \\ Andrea Bernardes 5 \\ Carmem Silvia Gabriel ${ }^{6}$
}

\section{Resumen}

Objetivo. Analizar las producciones científicas sobre la enseñanza del liderazgo en la graduación en Enfermería a partir del marco de las Directrices Curriculares Nacionales (DCN) de Brasil en 2001.

Método. Se realizó una revisión integradora de la literatura sobre las producciones científicas acerca de la enseñanza del liderazgo en la graduación en enfermería, a partir de las DCN.

Resultados. Los artículos fueran categorizados por afinidad de contenido, siendo constituidas las categorías de análisis: los desafíos

1. Aspirante al grado académico de Máster en el Programa Fundamental de la Escuela de Enfermería de Ribeirao Preto de

2. Aspirante al grado académico de Máster en el Programa Fundamental de la Escuela de Enfermería de Ribeirao Preto de la Universidad de Sao Paulo-USP.

email: jorgeluizrigobello@ig.com.br

3. Aspirante al grado académico de Máster en el Programa Fundamental de la Escuela de Enfermería de Ribeirao Preto de la Universidad de Sao Paulo-USP.

email: paulaluize@bol.com.br

4. Aspirante al grado académico de Máster en el Programa Fundamental de la Escuela de Enfermería de Ribeirao Preto de la Universidad de Sao Paulo-USP.

email: prislabl@gmail.com

5. Profesora, con grado académico de doctorado, en el Departamento de Enfermería General y Especializada de la Escuela de Enfermería de Ribeirao Preto de la Universidad de Sao Paulo- EERP/USP.

email: andreab@eerp.usp.br

6. Profesora, con grado académico de doctorado, en el Departamento de Enfermería General y Especializada de la Escuela de Enfermería de Ribeirao Preto de la Universidad de Sao Paulo- EERP/USP.

email: cgabirel@eerp.usp.br 
y dificultades en la enseñanza del liderazgo en la graduación de enfermería; la importancia de la enseñanza del liderazgo en la graduación de enfermería y la aplicación de teorías de liderazgo y metodologías de enseñanza en la graduación de enfermería para el desarrollo del liderazgo.

Palabras clave: educación, enseñanza, liderazgo, enfermería.

\title{
Analyzing the teaching of leadership in nursing education in Brazil.
}

\begin{abstract}
Objetive. The aim of this study was to analyze the scientific production produced on the teaching of leadership in nursing undergraduate courses from the framework of the National Curriculum Guidelines (NCG) of 2001 in Brazil.

Method. We conducted an integrative review of the literature on the scientific productions about teaching leadership in undergraduate nursing the DCN.

Results. Reading and critical analysis of articles allowed categorize them by affinity content, so that the following categories of analysis were established: Challenges and difficulties founded in nursing undergraduate education about leadership; The importance of nursing undergraduate education about leadership and application of leadership theories and teaching methodologies in nursing degree for the leadership development.
\end{abstract}

Keywords: education, teaching, leadership, nursing.

Recibido: 14-07-2015

Aceptado: 17-09-2015 


\section{Introducción}

La formación profesional de enfermería es orientada por el proceso histórico, político y social de una época en la cual se delinean los factores de configuración y estructuración de esta enseñanza, en cuya política pública se encuentra inseridos dichos factores (Renovato et al., 2009; Carvalho, 2011).

En este proceso, el currículo se presenta como el resultado de una selección de conocimientos y sabiduría que se desarrollan históricamente actuando como formas de poder y representación de sistemas de regulación y control, siendo un marco estructurante en la construcción de un nuevo paradigma en la educación (Germano, 2003; Renovato et al. 2009).

Así, las Directrices Curriculares Nacionales (DCN) se presentan, además de un marco histórico, como un apoyo para la creación de un nuevo paradigma en la educación de enfermería, articulando el conocimiento a las directrices del Sistema Único de Salud (SUS) y a las necesidades de la población (Teixeira et al., 2013).

Delante de esa coyuntura, se enfatiza que el concepto de habilidades se presenta como central en las propuestas de reformas curriculares en distintos niveles y modalidades de enseñanza de diferentes países. En Brasil, este concepto se constituye como una referencia para las directrices curriculares oficiales y para la evaluación de sistemas escolares.

Entre las habilidades necesarias para el ejercicio de la enfermería instituidas por las DCN, se destacan: la atención a la salud, la toma de decisiones, la comunicación, la administración y gestión, la educación permanente y el liderazgo, este último objeto del presente estudio.

La temática "habilidad profesional" se ha constituido, a lo largo de los años, en foco de atención de los enfermeros, así como de los administradores de los servicios de salud, ya que el personal de enfermería representa, en términos cuantitativos, una parte significativa de los recursos humanos destinados a esas instituciones, especialmente en los hospitales, y, por tanto, interfieren directamente en la eficacia, en la calidad y en el costo de la asistencia a la salud prestada. En ese sentido, la movilización de habilidades entre esos profesionales podrá reflejarse significativamente en los resultados obtenidos (Camelo, 2012).

La dimensión interpersonal y la habilidad humana del enfermero líder, es factor de motivación y soporte para el trabajo en equipo. La capacidad del líder de percibir, atender y estar conectado a las necesidades del equipo de trabajo, refuerza el sentimiento de pertenecer y nutre la autoestima de todos en el grupo, lo que conduce a alcanzar el objetivo de excelencia en la práctica clínica (Strapason, \& Medeiros, 2009). 
Delante del proceso histórico y de la reestructuración curricular en la enseñanza de enfermería a respecto de las habilidades, de la reflexión acerca de los desafíos y dificultades en la formación de los profesionales enfermeros en la enseñanza del liderazgo, así como del impacto de ésta para obtener una asistencia de calidad, se justifica la necesidad de la realización del presente estudio.

Así, se objetivó analizar las producciones científicas elaboradas sobre la enseñanza del liderazgo en los cursos de graduación en Enfermería a partir del marco de las Directrices Curriculares Nacionales (DCN) de 2001; identificar los modelos de liderazgo abordados en los cursos de graduación en Enfermería; analizar las reflexiones producidas sobre el perfil de la formación del profesional enfermero a partir de las DCNs; y analizar los desafíos y dificultades en la enseñanza del liderazgo en los cursos de graduación en Enfermería.

Para una adecuada reflexión frente a los objetivos propuestos, se utilizó como referencial teórico a las Directrices Nacionales Curriculares brasileñas para el curso de graduación en Enfermería.

En noviembre de 2001, el Consejo Nacional de Educación instituyó, por medio de la Resolución $\mathrm{n}^{\mathrm{o}}$ 3, las Directrices Nacionales Curriculares (DCN) del Curso de Graduación de Enfermería (Brasil, 2001a).

Las DCN constituyen una orientación para la elaboración de la matriz curricular del curso de graduación en enfermería, objetivando proveer al académico con los conocimientos necesarios para una formación sólida, dentro de una perspectiva que lo califique y lo prepare para enfrentar los desafíos y transformaciones impuestas por el mercado de trabajo y la sociedad.

Para esto, se objetiva desarrollar habilidades y capacidades, por medio de contenidos curriculares, prácticas y actividades complementarias, de acuerdo con el perfil epidemiológico en el cual el curso está inserido, debiendo el mismo poseer estructura y organización necesarias para un adecuado acompañamiento y evaluación, tanto del discente como del propio curso.

Esta Resolución enfatiza la necesidad de que exista articulación entre las Instituciones de Enseñanza Superior (IES) y la salud, guiándose por los principios del SUS para contener los objetivos de estas directrices que conducirán a “Aprender a aprender, lo que engloba aprender a ser, aprender a hacer, aprender a vivir juntos y aprender a conocer, garantizando la capacitación de profesionales con autonomía y discernimiento para asegurar la integralidad de la atención y la calidad y humanización de la atención prestada a los individuos, familias y comunidades" (Brasil, 2001,p.4).

En su artículo $4^{\circ}$, muestra la necesidad de preparar al profesional para asumir habilidades de atención de la salud, toma de decisiones, comunicación, liderazgo, 
administración y gestión, además de educación permanente, con la finalidad de que el graduado atienda a las necesidades sociales con énfasis en la calidad de una atención humanizada (Brasil, 2001b).

Además de eso, permiten que los currículos propuestos puedan construir el perfil académico y profesional con habilidades, capacidades y contenidos, dentro de perspectivas y abordajes contemporáneos de formación pertinentes y compatibles con referencias nacionales e internacionales, capaces de actuar con calidad, eficiencia y de forma resolutiva (Brasil, 2001b).

De acuerdo con las DCN, se espera que el enfermero obtenga una formación generalista, calificado para el ejercicio de la Enfermería, siendo capaz de conocer e intervenir en problemas y situaciones del proceso salud y enfermedad, además de actuar con sentido crítico y de responsabilidad, promoviendo la salud integral para el ser humano (Brasil, 2001b).

Las DCN definen los contenidos esenciales a ser adoptados en los cursos de graduación, incluyendo un abordaje teórico-práctico en los temas de ciencias biológicas, humanas y de enfermería, incluyendo la obligatoriedad de incluir la Práctica Curricular Supervisada (PCS) con un total mínimo de 20\% de la carga horaria del curso de graduación en Enfermería, la que será desarrollada en hospitales, ambulatorios y redes de salud, bajo la supervisión del profesor y del profesional enfermero del servicio (Brasil, 2001a, 2007).

La PCS tiene como principal objetivo la aproximación del alumno con la realidad práctica objetivando su interacción y aproximación con la profesión para que ésta sea un facilitador de su actuación después de la conclusión del curso de graduación.

Cabe destacar que la aproximación entre docente y alumno es necesaria, así como la responsabilidad del profesor en la articulación de la formación y en la creación de oportunidades para el desarrollo social del estudiante por medio de la educación problematizadora de carácter reflexivo, permitiendo que el estudiante desarrolle el sentido crítico entre su aprendizaje y su práctica (Alves, Burgatti, \& Oliveira, 2013).

El aprendizaje compatible con las DCN, así como el canal de comunicación eficaz entre docente y alumno durante el período de graduación, y el aprovechamiento de éste para el desarrollo del liderazgo, para actuar en equipos de trabajo conforme la necesidad de mercado, es una tarea obligatoria del profesional enfermero (Brasil, 2001a; Alves, Burgatti, \& Oliveira, 2013). 


\section{Metodología}

El presente estudio trata sobre la revisión integradora realizada en las siguientes etapas: definición del tema y pregunta orientadora; levantamiento de la literatura por medio de la selección cuidadosa de las investigaciones; categorización de las investigaciones encontradas; análisis crítico de los estudios incluidos; e interpretación de los resultados y análisis crítico frente a los resultados en comparaciones con otras investigaciones y síntesis evidenciadas en otras investigaciones (Cervo, \& Bervian, 2002).

Con la perspectiva de delinear una visión crítica y reflexiva acerca de la enseñanza del liderazgo en la graduación en enfermería, la pregunta orientadora fue: “¿Cuáles son las producciones cientificas elaboradas acerca de la enseñanza del liderazgo en la graduación en enfermería, a partir del marco de las Directrices Curriculares Nacionales (DCN) de 2001?”.

La investigación fue auxiliada por un levantamiento sistematizado de artículos en periódicos nacionales e internacionales, por medio de las bases de datos: Literatura Latinoamericana y del Caribe en Ciencias de la Salud (LILACS); Scientific Electronic Library Online (SCIELO); y PUBMED.

Los descriptores utilizados para seleccionar las publicaciones en las bases de datos LILACS y SCIELO fueron: "enfermería", "educación", "liderazgo". En la base de datos PUBMED fueron utilizados los descriptores Medical Subject Headings (MESH) compatibles con los descriptores de las demás bases de datos utilizadas, los cuales fueron: "education nursing", "leadership", "nursing" y "teaching".

Los artículos seleccionados y los demás documentos consultados fueron incluidos mediante los siguientes criterios: artículos publicados en el período de 2001 hasta el momento de la búsqueda que fue el año 2014 y artículos completos disponibles online en el idioma portugués encontrados por los descriptores ya mencionados. El criterio de inclusión de artículos apenas en el idioma portugués se debió a la necesidad de interrelacionar los temas frente a las DCN del curso de graduación en Enfermería en Brasil, las que orientaron el presente estudio.

Fueron criterios de exclusión: artículos que no estaban disponibles en su totalidad, libros y editoriales; habiéndose seguido el siguiente orden de búsqueda: LILACS, SCIELO y posteriormente PUBMED.

Los artículos para evaluación de inclusión en la investigación fueron sometidos al método de lectura científica compuesto por las siguientes etapas: lectura de reconocimiento, que objetivaba localizar en las fuentes una aproximación preliminar con el tema; lectura selectiva, en la cual se obtendría una visión de las informaciones de acuerdo con los objetivos del estudio; y finalmente, la 
lectura analítica correspondiendo a un carácter crítico-reflexivo de los textos seleccionados.

Esta última lectura fue acompañada de una reflexión contenida en una visión sintética, que constituye la última etapa del referido método que es consolidado a través de la lectura interpretativa (Cervo, \& Bervian, 2002).

Para el análisis y posterior síntesis de los artículos que atendieron a los criterios de inclusión fue utilizado un cuadro sinóptico especialmente construido para ese objetivo, el que contempló los siguientes aspectos, considerados pertinentes: objetivos, metodología, resultados relacionados con la temática del estudio y, posteriormente se elaboró la categorización de las mismas, interpretación de los datos y síntesis del conocimiento.

Esa categorización es utilizada en las Revisiones Integradoras para el análisis de los datos de una investigación cualitativa. Consiste en la reducción, exposición y comparación, así como en la conclusión y verificación de los datos. La reducción de los datos consiste en la determinación de un sistema de clasificación general para la administración de las diversas metodologías (Whittemore, 2005).

En principio, los estudios fueron divididos en subgrupos, de acuerdo con una clasificación establecida previamente, objetivando facilitar el análisis. La categorización se basó en la afinidad de contenidos y clasificaciones conceptuales determinadas posteriormente a la lectura de los estudios. A continuación, para facilitar la visualización estructural y lógica del estudio, se confeccionaron las fichas de lectura, con el objetivo de destacar las unidades de registro para agrupar los diferentes trabajos por temas.

Para la selección sistemática de los artículos objetivando el mayor número de artículos que pudiesen contemplar a los objetivos propuestos, los mismos fueron agrupados con los descriptores ya citados, en cada base de datos utilizada en este estudio, siguiendo el criterio de orden de búsqueda para una mejor evaluación crítica en caso de artículos repetidos.

Fueron encontrados 363 resúmenes, de acuerdo con la suma de los artículos de todas las bases de datos investigadas, para el análisis inicial de inclusión o exclusión y, posteriormente, fueron seleccionados para el análisis del estudio 52 artículos (14,33\%), estando los mismos adecuados con los criterios de inclusión. Después de esta etapa, se procedió a una nueva selección de los artículos buscando, con eso, evitar la repetición de estudios en las bases de datos, así fueron excluidos $42(80,77 \%)$ artículos.

Para contemplar un número mayor de artículos se realizó una búsqueda seguida de una lectura cuidadosa y exhaustiva, incluyendo diferentes formas de los descriptores para el levantamiento bibliográfico, además de la palabra clave 
"Educación”. Siguiéndose todos los criterios de selección y excluyéndose los artículos repetidos, se observó que, en la base de datos PUBMED, todos los estudios seleccionados ya habían sido contemplados en las otras bases de datos, resultando, así, en un total de 10 artículos $(2,75 \%)$ a ser analizados.

El análisis crítico de los artículos fue realizado mapeando sus objetivos, metodología, resultados relacionados con la temática del estudio; posteriormente, se elaboró la categorización de los mismos, interpretación de los datos y síntesis del conocimiento.

\section{Resultados}

Después de la lectura intensiva y del análisis crítico de los relatos de los 10 artículos, los datos fueron clasificados en unidades de registro para llegar al núcleo o significado del texto. Después, fueron creadas tres categorías de análisis: desafíos y dificultades en la enseñanza del liderazgo en la graduación de enfermería, con 5 artículos (50\%); importancia de la enseñanza del liderazgo en la graduación de enfermería y aplicación de teorías de liderazgo, con 3 artículos (30\%); y metodologías de enseñanza en la graduación de enfermería para el de desarrollo del liderazgo, con 3 artículos (20\%).

En relación a la metodología utilizada, hubo el predominio de estudios del tipo descriptivo con abordaje cualitativo, en 6 artículos (60\%), 3 estudios reflexivos $(30 \%)$ y 1 artículo con abordaje tanto cualitativo como cuantitativo (10\%). En lo que se refiere al local de la publicación, $100 \%$ de los trabajos fueron publicados en periódicos de enfermería nacionales.

Para la categorización de los temas, se adoptó a estrategia de la subdivisión en tres grupos de semejanza, conforme presentados a seguir.

\section{La importancia de la enseñanza del liderazgo en la graduación de enfermería}

Los tres artículos contemplados en esta categoría abordan la importancia de la enseñanza del liderazgo en la graduación de enfermería.

Entre los trabajos aparece un estudio sobre el significado del liderazgo para el graduando en enfermería, destacando que el liderazgo es una de las principales habilidades a ser adquirida por el enfermero, siendo esa afirmación corroborada por las DCN, que resaltan ser necesaria la formación de un profesional con sentido crítico, capaz de resolver problemas y desarrollar la habilidad del liderazgo (Brasil, 2001a, Guerra, \& Spiri, 2013).

La enseñanza del liderazgo para el graduando de enfermería posee un papel fundamental en el engranaje de una institución de salud, sea esta en ambiente 
hospitalario o en unidad básica de salud, ya que busca desarrollar y perfeccionar habilidades que favorezcan la conducción equilibrada de un grupo, entre ellas la búsqueda constante por el conocimiento, la comunicación eficiente, la capacidad resolutiva y el buen relacionamiento interpersonal; habilidades que proporcionan, en el ejercicio del liderazgo, un ambiente favorable para la ejecución del cuidado, asumiendo la difícil tarea de coordinar un equipo de enfermería (Gaidzinski, Peres, \& Fernandes, 2004).

Así, de acuerdo con los autores arriba mencionados, el liderazgo requiere cualidades individuales, organizacionales y grupales que pueden ser aprendidas y desarrolladas. Como, también, el aprendizaje continuo del liderazgo requiere ambientes organizacionales en donde los trabajadores deben aprender a asumir riesgos, a salir de posiciones confortables y a experimentar nuevas ideas y experiencias.

Guerra \& Spiri (2013) realizaron un estudio cualitativo - que objetivó comprender el significado del liderazgo para el estudiante de enfermería y la expectativa relacionada a su práctica profesional - con 15 alumnos que cursaban el sétimo período de un curso de graduación de Enfermería. Los alumnos relataron diferentes formas de liderazgo de acuerdo con la situación, siendo estas influenciadas por la política institucional, la enseñanza y el liderado. Los alumnos enfatizaron la dicotomía entre la teoría y la práctica del liderazgo, debiendo realizarse esfuerzos conjuntos por parte de las Instituciones de Enseñanza Superior (IES) y de los servicios, para proporcionar una mejor formación crítica y reflexiva y para promover cambios sociales y en la atención a la salud. Por medio del recorte fenomenológico, se comprendió que el significado atribuido al liderazgo es revelado como un proceso dinámico y que el estilo adoptado es la forma de conducir un equipo, no existiendo un estilo ideal de liderazgo. En la enseñanza, el estilo de liderazgo pasó a ser discutido cuando se abordaron las formas participativas de administración de personal. En el Ejercicio del Liderazgo, es enfatizada la disociación de la teoría con la práctica del liderazgo.

Así, el conocimiento teórico es esencial para el desarrollo del liderazgo, evidenciando que para el desarrollo de un liderazgo eficaz es necesaria, la combinación del aprender a conocer, aprender a hacer, aprender a vivir juntos y aprender a ser (Guerra, \& Spiri, 2013).

Además de eso, la formación de enfermeros-líderes se impone como una gran necesidad, ya que en el escenario actual se buscan, cada vez más, profesionales competentes técnicamente y científicamente, y capaces de administrar el cuidado y el equipo de enfermería, utilizándose el diálogo, la ética y la humanización (Coutinho; Souza; Ferreira, 2002).

En un estudio realizado por los autores, arriba mencionados, se destaca que el "proceso de enseñanza-aprendizaje del liderazgo aún se manifiesta como un 
desafío, no apenas para el proceso formativo, pero también para los servicios de salud y para enfermeros, ya que se trata de una condición esencial, la cual posibilita la visualización de un futuro con nuevas perspectivas y propuestas de actuación, pudiendo contribuir para una mayor visibilidad y valorización de la profesión".

Así, se entiende que la formación del enfermero-líder posibilita, a este profesional, tornarse un agente de cambios, creando innovaciones con el propósito de mejorar la organización, el equipo de enfermería y principalmente la asistencia prestada al paciente.

\section{Metodologías de enseñanza en la graduación para o de desarrollo del liderazgo}

Los dos trabajos encontrados $(20 \%)$ reflexionan sobre las metodologías de enseñanza en la graduación para el desarrollo del liderazgo, relatando la necesidad de la implementación de estrategias pedagógicas más activas e innovadoras en la enseñanza del liderazgo, utilizando nuevos instrumentos pedagógicos que permitan alcanzar habilidades importantes para el ejercicio académico y profesional del enfermero (Costantino, \& Sanna, 2006; Ramos, Freitas, \& Silva, 2011; Cotta, Costa, \& Mendonça, 2013).

El estudio realizado por Cotta, Costa, \& Mendonça (2013) demostró que la utilización del portafolio de evidencias auxilia en el desarrollo de futuros profesionales de la salud de una forma reflexiva a través de la construcción de la autonomía, compromiso con el trabajo y desarrollo de liderazgo, habilidades estas esenciales para el trabajo. Siendo que, el mismo fue considerado un facilitador en la reconstrucción del aprendizaje del liderazgo como habilidad profesional.

En esa perspectiva, el portafolio de evidencias representa una herramienta útil no solo como instrumento de evaluación del desempeño, pero también como estrategia para estimular el aprendizaje centrado en habilidades, actitudes y conocimiento, el "aprender haciendo". En este aspecto, el uso del portafolio es un método que proporciona un proceso de enseñanza-aprendizaje activo, cuyo enfoque metodológico se basa en la comunicación dialógica entre los diferentes sujetos, que pretende que los estudiantes desarrollen habilidades, como el liderazgo (Cotta, Costa, \& Mendonça, 2013).

En otro estudio sobre la contribución de nuevas metodologías en el aprendizaje del liderazgo, se observó que para el desarrollo de esa habilidad, es necesario hacer inversiones en la formación del enfermero-líder, lo que posibilita que éste profesional se torne un agente de cambios, contribuyendo con innovaciones para la mejoría de la asistencia prestada al individuo, familia y comunidad, y es en este escenario de formación que se destacó el Internado en Enfermería, el cual proporciona una vivencia integral en el campo asistencial de salud, que conduce 
a la mejoría del proceso enseñanza-aprendizaje de los futuros enfermeros (Ramos, Freitas, \& Silva, 2011).

Además de eso, ese mismo estudio afirma que el internado fue una herramienta de aprendizaje del liderazgo que proporcionó una nueva metodología de enseñanza-aprendizaje, posibilitando el contacto más prolongado del estudiante de enfermería con la institución de salud, con los profesionales, con los pacientes y con los familiares. Partiendo de la premisa de que la habilidad en liderazgo es posible de ser aprendida y que las experiencias son fundamentales en la concretización de este conocimiento, se identificó que el internado contribuyó de forma decisiva para el aprendizaje del liderazgo de los estudiantes de enfermería de una universidad estatal del estado de Ceará (Ramos, Freitas, \& Silva, 2011).

\section{Desafíos y dificultades en la enseñanza del liderazgo en la graduación de Enfermería}

En relación a los desafíos y dificultades en la enseñanza del liderazgo en la graduación de enfermería fueron encontrados cuatro artículos contemplados en esa categoría.

Amestoy et al. (2013) buscando conocer la percepción de los enfermeros sobre el proceso de enseñanza-aprendizaje del liderazgo, identificó que la formación académica está distante del desarrollo de habilidades que auxilian el estudiante a liderar.

Se relató también, el énfasis dado al tecnicismo, o sea, la preocupación del alumno en realizar procedimientos técnicos durante la graduación y el alejamiento de las actividades administrativas.

Se verificó además que los docentes poseen dificultades en adherir a las nuevas prácticas pedagógicas, como las metodologías activas, y hasta cierta falta de preparación para utilizarlas, lo que limita el establecimiento de una relación dialógica y constructiva entre los sujetos cognoscibles, esto es, entre el educador y el estudiante (Peres, \& Ciampone, 2006; Amestoy, et al., 2013).

En otro estudio sobre el papel de una institución de enseñanza superior en la formación de enfermeros-líderes, fueron encontradas declaraciones de enfermeros que expresaban la existencia de vacíos en el proceso de enseñanzaaprendizaje del liderazgo durante la graduación, vacíos estos producidos por el poco tiempo utilizado en la enseñanza de esa habilidad y el distanciamiento de los docentes de la práctica asistencial (Amestoy et al., 2010).

Además, sobre los desafíos en la enseñanza del liderazgo, los investigadores relatan que los contenidos teóricos han sido presentados en la forma de clases exhaustivas, sin la debida relevancia de su significado real y desarticulados entre 
sí, no ofreciendo el retorno deseado por el alumno. Este tipo de enseñanza puede explicar la dificultad en el ejercicio del liderazgo, teniendo en consideración que el mismo, cuando descontextualizado de la práctica y de la realidad laboral, no instrumentaliza al futuro profesional para ser líder del equipo, una vez que el liderazgo exige tomar decisiones a partir de lo vivido (Amestoy et al., 2010).

Según Vilela \& Souza (2010) uno de los motivos que contribuyen para la dificultad del enfermero en ejercer el liderazgo es la insuficiencia en la formación profesional para alcanzar esta habilidad, siendo necesario que las instituciones de enseñanza valoricen este contenido temático, articulando la teoría con la práctica en la formación académica de los enfermeros, con el objetivo de prepararlos para el ejercicio del liderazgo, que es una de las habilidades exigidas por el mercado de trabajo.

Constantino \& Sanna (2006) percibiendo la existencia de vacíos en el proceso educacional sobre las diversas formas de desarrollar el liderazgo en la graduación de enfermería y las autoras de otro estudio, al analizar los planes de enseñanza, encontraron una desvalorización de la enseñanza del Liderazgo por los docentes, pudiendo afirmarse que existe un descompás entre lo que se propone y lo que se efectiva, ya que el liderazgo parece no ser lo que se pretendía enseñar a los alumnos.

De esa forma, se concluye que la importancia de la formación de enfermeroslíderes es innegable, ya que el liderazgo es una habilidad inherente a este profesional. Sin embargo, se constata que los enfermeros están poco instrumentalizados para ejercer el liderazgo en la práctica. Delante de las muchas habilidades necesarias que necesita el enfermero para su práctica profesional, se constata que desarrollarlas constituye un gran desafío, tanto de los órganos formadores como de los servicios, en la perspectiva de la educación permanente, y se torna una responsabilidad de todos estos actores: de los docentes, de los enfermeros del servicio y del propio aprendiz.

\section{Conclusiones}

El presente estudio demostró que la habilidad del Liderazgo viene siendo tratada, por los responsables de la confección de matrices curriculares de los cursos de graduación en enfermería, de forma superficial, ya que esa temática es abordada de forma incipiente en algunas disciplinas, especialmente en las que se refieren a la Administración y Gestión de Enfermería, disciplina ésta desarrollada mayoritariamente en el último año del curso.

Se cree que con la falta de una disciplina específica (o hasta mismo la inclusión del tema de forma concreta, siguiendo una línea transversal en la matriz curricular) la formación del futuro profesional quedará perjudicada, ya que 
cuando éste entre en el mercado de trabajo se espera que desarrolle eficazmente y efectivamente esa habilidad.

A pesar de que la característica del Liderazgo puede estar presente intrínsecamente en algunos individuos, los estudios demuestran que ella debe ser desarrollada y trabajada por las instituciones formadoras, buscando de esta forma un profesional más calificado.

Para se conseguir éxito en esa empresa es necesario realizar cambios que van desde las estrategias de las clases - en su mayoría expositivas de carácter depositario, a veces mantenedoras de la dicotomía entre la teoría y la práctica posible de ser amenizada por la adopción de la Integración Docente Asistencial - hasta una reestructuración del Proyecto Político Pedagógico del curso y de la IES.

Inclusive cuando el mercado de trabajo continúe siendo la principal influencia en la formación profesional, se espera que los cursos proporcionen la adquisición de conocimientos capaces de transformar al individuo en un ser reflexivo y crítico frente a la situación de la asistencia de la salud en el país, que sería capaz de contemplar tanto las necesidades de las instituciones de salud como las de usuarios de estos servicios.

Se encuentra ahí uno de los principales desafíos para las instituciones formadoras, ya que la tendencia cada vez mayor por atender solamente lo que el mercado necesita, lleva a un distanciamiento de lo que realmente está preconizado por las DCN para el curso de graduación en enfermería, que es garantizar una formación sólida para el futuro profesional guiada por la ética, por el conocimiento y por el ejercicio de la ciudadanía frente a las situaciones de asistencia de la salud de la población.

Los estudios revelaron que los principales participantes en el proceso enseñanzaaprendizaje en la graduación en enfermería: alumnos, docentes y alumnos egresados (profesionales), sienten la necesidad de obtener un futuro profesional mejor calificado para el desarrollo pleno de la habilidad del liderazgo, debiendo característica ser contemplada en la graduación, ya que solamente así se podrá tener un profesional más preparado para asistir mejor al usuario.

Los autores del presente estudio entienden que es necesario desarrollar más investigaciones en relación el tema aquí presentado, ya que la cantidad de artículos encontrados fue pequeña frente a lo que se produce en otros países.

Los mismos creen que con el desarrollo de más estudios, es posible sensibilizar a los responsables por la formulación de matrices curriculares de los cursos de graduación en Enfermería y de los administradores de las IES, en lo que se refiere a la necesidad de enseñar el Liderazgo en la formación del futuro Enfermero, en 
una disciplina específica o de modo más concreto y efectivo transversalmente en el núcleo de las disciplinas a lo largo del curso y no solamente en una única disciplina, de modo superficial, al final del curso.

\section{Referencias bibliográficas}

Alves, E., Burgatti, J. \& Oliveira, M. 2013. O pensamento crítico e reflexivo: percepção de docentes de um currículo integrado. Indagatio Didactica, Universidade de Aveiro, Portugal, 5(2).

Amestoy, S. et al. 2010. Processo de formação de enfermeiros líderes. Rev. Bras. De Enfermagem, Brasília, 63(6).

Amestoy, S. et al. 2013. Percepção dos enfermeiros sobre o processo de ensinoaprendizagem da liderança. Rev.Texto Contexto enferm, 22(2), pp. 468-75).

Brasil. 2001a. Conselho Nacional da Educação/Câmara de Educação Superior. Resolução CNE/CES 3/2001 de 07 de Novembro de 2001. Institui as Diretrizes Nacionais Curriculares do Curso de Graduação de Enfermagem. Diário Oficial da União, Brasília:Ministério da Educação e Cultura, Seção 1, p. 37

Brasil. 2001b. Conselho Nacional da Educação/Câmara de Educação Superior. Parecer CNE/CES 1133/2001, homologado em 07 de agosto de 2001. Dispõe sobre as Diretrizes Nacionais Curriculares dos Cursos de Graduação em Enfermagem, Medicina e Nutrição. Diário Oficial da União, Brasília, Seção 1E, p. 131.

Brasil. 2007. Conselho Nacional da Educação/Câmara de Educação Superior Resolução CNE/CES n. 33/2007 de 01 de fevereiro de 2007. Consulta sobre a carga horária do curso de graduação de enfermagem e sobre a inclusão do percentual destinado ao Estágio Supervisionado na mesma carga horária. Diário Oficial da União, Brasília.

Camelo, S. 2012. Professional competences of nurse to work in Intensive Care Units: an integrative review. Rev. Latino-Am. Enfermagem, Ribeirão Preto, 20(1).

Carvalho, L .2011. Uma antiga profissão do futuro: percepções de enfermeiros sobre sua formação e inserção profissional. Dissertação de mestrado não-publicada, Escola Nacional de Saúde Pública Sergio Arouca, Rio de Janeiro, Brasil.

Cervo A. \& Bervian, P. 2002. Metodologia Científica, São Paulo:PrenticeHall.

Constantino, L. \& Sanna, M. 2006. A inserção do tema Liderança nas disciplinas de Administração em Enfermagem de uma faculdade particular da cidade de São Paulo 1998 a 2004, Esc. Anna Nery, Rio de Janeiro, 10(2). 


\section{Revista de}

Cotta, R.; Costa, G. \& Mendonça, E. 2013. Portfólio reflexivo: uma proposta de ensino e aprendizagem orientada por competências. Ciência \& Saúde Coletiva, Rio de Janeiro, 18(6).

Coutinho, R.; Souza, N. \& Ferreira, H. 2002. A necessidade da liderança na formação do enfermeiro do século XXI. Revista SOBECC, São Paulo, 7(4), pp.25-30.

Gaidzinski, R.; Peres, H. \& Fernandes, M. 2004. Liderança: aprendizado contínuo no gerenciamento em enfermagem. Rev. bras. Enferm, Brasília, 57(4).

Guerra, K. \& Sipri, W. 2013. Compreendendo significado da liderança para o aluno de graduação em enfermagem: uma abordagem fenomenológica. Rev. bras. Enferm, Brasília, 66(3), pp. 399-405.

Peres, A. \& Ciampone, H. 2006. Gerência e competências gerais do enfermeiro. Rev. Texto contexto enferm, 15(3), pp. 492-99.

Ramos, V.; Freitas, C. \& Silva, M. 2011. Aprendizagem da liderança: contribuições do internato em enfermagem para formação do estudante. Esc. Anna Nery, Rio de Janeiro,15(1).

Renovato, R.; Bagnato, M.; Missio L. \& Bassinello, G. 2009. As identidades dos enfermeiros em cenários de mudanças curriculares no ensino da enfermagem. Rev.Trab. Educ. Saúde, Rio de Janeiro, 7 (2), 231-248.

Strapasson, M. \& Medeiros, C. 2009. Liderança transformacional na enfermagem. Rev. bras. Enferm, Brasília, 62(2), pp. 228-33.

Teixeira, E.; et al. 2013. Panorama dos cursos de Graduação em Enfermagem no Brasil na década das Diretrizes Curriculares Nacionais. Rev Bras Enferm, Brasília (DF), 66, pp. 102-10.

Vilela, P. \& Souza, A. 2010. Liderança: um desafio para o enfermeiro recém-formado, Revista de Enfermagem da Universidade Estadual do Rio de Janeiro, Rio de Janeiro, 18(4), pp.591-97.

Whittemore. \& Knafl, R. 2005. The integrative review: update methodology. Journal of Advanced Nursing, 52(5), pp.456-553. 